\title{
FATORES DE RISCO ASSOCIADOS À PRESENÇA DE ANTICORPOS ANTILEPTOSPIRA EM TRABALHADORES DO SERVIÇO DE SANEAMENTO AMBIENTAL ${ }^{1}$
}

\author{
RISK FACTORS ASSOCIATED TO THE PRESENCE OF ANTIBODIES \\ ANTI-LEPTOSPIRA IN ENVIRONMENTAL SANITATION WORKERS
}

\author{
Laerte Pereira de Almeida ${ }^{2}$ Luis Fernando da Silva Martins ${ }^{3}$ Claudiomar Soares Brod ${ }^{4}$
}

RESUMO

Realizou-se um estudo soroepidemiológico com trabalhadores do Saneamento Ambiental de Pelotas-RS para estimar a freqüência e investigar fatores de risco associados à infecção por leptospira. A partir do resultado sorológico, selecionaram-se 2 grupos de trabalhadores, divididos em um grupo de 34 indivíduos com sorologia positiva, casos; e um grupo de $115 \mathrm{com}$ sorologia negativa, controles. Através de um questionário padronizado, coletaram-se informações sobre variáveis demográficas, ocupacionais, comportamentais e ambientais nos locais de trabalho e de moradia dos indivíduos. A freqüência de aglutininas anti-leptospira foi igual a 10,36\% $(n=386)$. Os fatores de risco detectados foram: local geográfico de trabalho; presença de esgotos a céu aberto, criação de suínos e presença de 3-4 animais no local de moradia e, como fator de proteção, a presença de sanitário interno à moradia. Essas informações são úteis para prevenir a leptospirose nessa categoria de trabalhadores.

Palavras-chave: leptospirose, saneamento ambiental, zoonoses, epidemiologia.

\section{SUMMARY}

A seroepidemiological survey was realized with environmental sanitation workers of Pelotas-RS city, Brazil, to examine the frequency and to investigate risk factors for leptospira infection. Two groups of workers were selected due to serological status, divided into groups of 34 subjects with positive serology, cases; and a group of 115 subjects with negative serology, the later one being the control group. Demografics variables, occupational, behaviors and environmental in work places and home were investigated by a questionaire. The frequency of anti-leptospiral agglutinins was $10.36 \%(n=386)$.
The risk factors detected were: geografic place of work, the presence of oppened drain, swine creation and the presence of 34 animals at home, and the protection factor in the presence of internal sanitation of residence. This information may be useful to prevent leptospirosis in environmental sanitation workers.

Key words: leptospirosis, environmental sanitation, zoonosis, epidemiology.

\section{INTRODUÇÃO}

$\mathrm{Na}$ atualidade, tanto em países industrializados quanto em países em desenvolvimento, um considerável número de zoonoses tem emergido ou como uma nova entidade patológica ou como agentes já conhecidos, aparecendo em áreas ou espécies que ainda não tinham sido registradas. Adicionalmente, alguns agentes de zoonoses já conhecidos têm reemergido após muitos anos ausentes de determinadas áreas. Entre as zoonoses reemergentes têm sido assinaladas a raiva, brucelose, anthrax e leptospirose, e, como zoonoses emergentes, algumas doenças veiculadas por alimentos e arboviroses com o envolvimento de produtos de origem animal (MESLIN, 1997).

RATHINAM et $\boldsymbol{a l}$. (1997) têm descrito a leptospirose como doença reemergente, cuja ocorrência, na forma de surtos em humanos ou animais, tem sido registrada em vários países associada a

\footnotetext{
${ }^{1}$ Extrato de Dissertação de Mestrado intitulada "Estudo retrospectivo de reservatórios animais e vias de transmissão para leptospirose em cinco categorias de trabalhadores. Pelotas-RS" apresentada à Universidade Federal de Pelotas, RS.

${ }^{2}$ Médico Veterinário, Doutor., Professor Adjunto, Departamento de Nutrição e Saúde, Universidade Federal de Viçosa (UFV) Avenida PH Rolfs, s/n, Campus Universitário, 36571-000, Viçosa, Minas Gerais. E-mail:lalmeida@mail.ufv.br. Autor para correspondência.

${ }^{3}$ Médico Veterinário, MSc., Professor Departamento de Veterinária da Universidade Federal de Pelotas.

${ }^{4}$ Médico Veterinário, Especialista, Professor do Departamento de Veterinária da Universidade Federal de Pelotas. 
diferentes circunstâncias, prática de esportes e lazer (KATZ et al., 1997), em atividades ocupacionais (PERROCHEAU \& PEROLAT, 1997) ou, apresentando sintomatologia diferenciada da forma clássica descrita (ZARI \& SHIEH, 1996).

A leptospirose, classicamente, tem sido associada a determinadas categorias ocupacionais como, trabalhadores em abatedouros, tratadores de animais, médicos veterinários e saneamento ambiental (ACHA \& SZYFRES 1986). Apesar disso, alguns autores têm sugerido o envolvimento de variáveis sociais na ocorrência da doença (EVERARD $\boldsymbol{e} t$ al.1995; ANDRADE \& BRANDÃO, 1987).

O conhecimento da freqüência de infecção antileptospira e de seus determinantes em populações de trabalhadores pode ser de grande valor para orientar as ações preventivas de saúde. Neste sentido, planejou-se um estudo soroepidemiológico com trabalhadores do Serviço de Saneamento Ambiental de Pelotas, RS, com o objetivo de identificar a frequiência de anticorpos antileptospira e sua associação com fatores de risco, avaliados nos locais de trabalho e de moradia.

\section{MATERIAL E MÉTODOS}

Os dados foram obtidos como parte de um estudo sobre leptospirose em trabalhadores do Serviço de Saneamento Ambiental da cidade de Pelotas, Rio Grande do Sul. Parte da metodologia, técnica diagnóstica e resultados sorológicos já foram descritos em trabalho anterior (ALMEIDA et al. 1994; ALMEIDA, 1991).

Foram investigados 386 indivíduos $(71 \%$ do efetivo de trabalhadores sob a condição pesquisada) dos quais se colheram amostras de sangue e informações sobre variáveis ocupacionais, comportamentais e ambientais nos locais de trabalho e moradia através de um questionário padronizado, précodificado e testado (ALMEIDA et al.,1994).

Para a verificação da presença de fatores de risco associados com anticorpos antileptospira, focalizou-se um grupo de trabalhadores, testados sorologicamente no estudo, divididos em um grupo de 34 indivíduos com resultado sorológico positivo para a infecção, título de anticorpos maior ou igual a 100 (casos) e um grupo de 115 indivíduos com sorologia negativa, título de anticorpos menor que 25 (controles).

Os dados coletados foram armazenados em banco de dados, criado através do software Epi Info 6.04. Para a análise, inicialmente, buscou-se verificar a existência de associações significativas entre as variáveis estudadas e a presença de anticorpos antileptospira. Estas associações foram avaliadas através do cálculo da razão de "odds", de seu intervalo de confiança de $95 \%$ e do teste do Quiquadrado $\left(\mathrm{X}^{2}\right)$, (KIRKWOOD, 1988).

Para medir o efeito de variáveis que se mostraram associadas, na análise bivariada, com anticorpos antileptospira, foi realizada análise de regressão logística, não condicional, por meio do programa Multlr. As medidas de "odds ratio", obtidas por este procedimento, serão referidas como "odds ratio" ajustadas.

\section{RESULTADOS}

O exame de 386 soros de trabalhadores do Serviço de Saneamento Ambiental evidenciou 40 indivíduos com títulos de anticorpos antileptospira maior ou igual a 100 , considerado positivo para a infecção, o que equivale a uma freqüência igual a $10,36 \%$.

Quanto às características ocupacionais, a média de antiguidade no trabalho mostrou 9,0 anos para órgão de trabalho $(\mathrm{s}=6,1)$ e 7,6 anos no setor $(\mathrm{s}=5,7)$. Destes, $82,6 \%$ não costumavam mudar de setor de trabalho e $91,9 \%$ disseram não terem recebido treinamento anterior. No desempenho de suas atividades, $47,7 \%$ dos trabalhadores referiram o uso de luvas, $55,0 \%$ o uso de botas de borracha e apenas 26,2\% o uso de macacão. Quanto ao local geográfico, onde suas atividades são desenvolvidas, 10,7\% trabalham no centro da cidade, $21,5 \%$ em um único bairro, $24,8 \%$ em vários bairros e $43,0 \%$ em toda a cidade.

As tabelas 1 a 3 mostram variáveis ocupacionais, ambientais no local de moradia e comportamentais, respectivamente. Em cada uma dessas tabelas estão dispostos: o valor da Razão de "odds" e intervalos de confiança (95\%), considerando como categoria de base aquela em que se esperava o menor risco e também a significância estatística da associação entre a variável estudada e a presença de anticorpos antileptospira.

Quanto às variáveis ocupacionais avaliadas, (análise bivariada, tabela 1) sobressaiu-se o local geográfico de trabalho $(\mathrm{OR}=3,12)$, mostrando que indivíduos com atividades em toda a cidade apresentam um risco 3 vezes maior em possuir anticorpos antileptospira do que aqueles com atividades em um único bairro ou no centro da cidade. Da mesma forma, trabalhadores que desenvolvem atividades em locais onde há a presença de gatos $(\mathrm{OR}=4,32)$ e de terrenos baldios $(\mathrm{OR}=2,77)$ apresentaram maior risco de sofrerem infecção por leptospira. Ainda, nesta categoria, aqueles indivíduos que no desempenho de suas funções tenham, de alguma forma, que lidarem com lixo doméstico ou 
Tabela 1 - Fatores de risco (Variáveis Ocupacionais) para infecção por leptospira. Pelotas/RS, Brasil. 1991.

\begin{tabular}{|c|c|c|c|}
\hline Variáveis & Razão de Odds (bruta) e IC* $95 \%$ & Número de Indivíduos & $\mathrm{P}$ \\
\hline Presença de gatos $* *$ & & & $=0,02$ \\
\hline Sim & $4,32(1,42-13,10)$ & 103 & \\
\hline Não & 1,00 & 43 & \\
\hline Contato com animais & & & $=0,7$ \\
\hline Sim & $1,26(0,37-4,25)$ & 15 & \\
\hline Não & 1,00 & 134 & \\
\hline Lidar com cães & & & $=0,07$ \\
\hline Sim & $3,13(0,89-10,99)$ & 11 & \\
\hline Não & 1,00 & 138 & \\
\hline Lidar com água parada & & & $=0,1$ \\
\hline Sim & $2,01(0,84-4,84)$ & 97 & \\
\hline Não & 1,00 & 52 & \\
\hline Desentupir bueiros & & & $=0,3$ \\
\hline Sim & $1,49(0,64-3,43)$ & 52 & \\
\hline Não & 1,00 & 97 & \\
\hline Lidar com lixo de rua, entulho & & & $=0,3$ \\
\hline Sim & $1,54(0,67-3,53)$ & 94 & \\
\hline Não & 1,00 & 55 & \\
\hline Lidar com lixo doméstico & & & $=0,07$ \\
\hline Sim & $1,93(0,87-4,27)$ & 78 & \\
\hline Não & 1,00 & 71 & \\
\hline \multicolumn{4}{|l|}{ Local geográfico de trabalho** } \\
\hline Centro/único bairro & 1,00 & 49 & \\
\hline Conjunto de bairros & $1,75(0,65-4,74)$ & 62 & \\
\hline Cidade toda & $3,12(1,10-8,86)$ & 38 & $=0,02$ \\
\hline Presença de terrenos baldios** & & & $=0,05$ \\
\hline Sim & $2,77(0,90-8,50)$ & 114 & \\
\hline Não & 1,00 & 35 & \\
\hline
\end{tabular}

* IC = intervalo de confiança.

***local onde são desempenhadas as atividades ocupacionais.

com cães, mostraram risco aumentado, embora com nível de significância limítrofe.

$\mathrm{Na}$ tabela 2, observa-se que, aqueles indivíduos que referiram possuírem criação de suínos no ambiente de moradia mostraram uma probabilidade 5 vezes maior de apresentarem anticorpos reagentes a leptospira $(\mathrm{OR}=4,98)$, enquanto que trabalhadores com 3 a 4 animais, neste ambiente, têm um risco 4 vezes maior $(\mathrm{OR}=3,94)$. Sobressaiu-se, nessa tabela, a presença de esgotos a céu aberto, onde moradores de ambientes com essa condição estariam 2,5 vezes mais em risco de infecção por leptospira do que moradores de localidades sem essa condição. Chama a atenção, ainda, em relação ao ambiente de moradia, por apresentar-se como fator de proteção aos trabalhadores contra a infecção por leptospira, a presença de banheiro interno à moradia $(\mathrm{OR}=0,33)$.

Em relação às variáveis comportamentais, não foi possível detectar nenhuma associação entre as variáveis avaliadas e a leptospirose, embora indivíduos identificados como tratadores de seus animais tenham demonstrado um risco da ordem de 2,5 vezes maior para a infeção.

$\mathrm{Na}$ análise multivariada, algumas variáveis perderam sua significância estatística e outras, praticamente, não sofreram alterações dos valores obtidos na análise bivariada. Permaneceu, após ajuste, como fator de proteção contra a infeção por leptospira, a presença de banheiro interno à moradia $(\mathrm{p}=0,02)$ e, como fatores de risco, a criação de suí- 
Tabela 2 - Fatores de risco (Variáveis Ambientais no local de Moradia) para a infecção por leptospira. Pelotas/RS, Brasil. 1991.

\begin{tabular}{|c|c|c|c|}
\hline Variáveis & Razão de Odds (bruta) e IC 95\% & Número de Indivíduos & $\mathrm{P}$ \\
\hline Presença de animal em casa & & & $=0,1$ \\
\hline Sim & $2,14(0,76-6,02)$ & 113 & \\
\hline Não & 1,00 & 36 & \\
\hline Presença de cães em casa & & & $=0,5$ \\
\hline Sim & $1,67(0,69-4,03)$ & 102 & \\
\hline Não & 1,00 & 47 & \\
\hline Presença de gatos em casa & & & $=0,7$ \\
\hline Sim & $1,09(0,64-1,86)$ & 50 & \\
\hline Não & 1,00 & 99 & \\
\hline Criação de suínos & & & $=0,02$ \\
\hline Sim & $4,98(1,06-23,46)$ & 7 & \\
\hline Não & 1,00 & 142 & \\
\hline Presença de ratos próximo à casa & & & $=0,1$ \\
\hline Sim & $2,03(0,93-4,44)$ & 72 & \\
\hline Não & 1,00 & 77 & \\
\hline Número de animais em casa & & & $=0,03$ \\
\hline Nenhum & 1,00 & 36 & \\
\hline 1-2 animais & $1,96(0,67-5,74)$ & 82 & \\
\hline 3-4 animais & $3,94(1,08-14,3)$ & 21 & \\
\hline 5 ou mais & $1,60(0,26-9,81)$ & 10 & \\
\hline Presença de terreno baldio, matas & & & $=0,4$ \\
\hline Sim & $1,32(0,61-2,83)$ & 71 & \\
\hline Não & 1,00 & 78 & \\
\hline O banheiro é interno à casa & & & $=0,008$ \\
\hline Sim & $0,33(0,14-0,75)$ & 39 & \\
\hline Não & 1,00 & 110 & \\
\hline Presença de esgotos a céu aberto & & & $=0,03$ \\
\hline Sim & $2,45(1,05-5,72)$ & 88 & \\
\hline Não & 1,00 & 61 & \\
\hline
\end{tabular}

nos $(\mathrm{p}=0,02)$ e presença de esgotos a céu aberto $(\mathrm{p}=0,04)$ no ambiente de moradia. Ainda, com nível de significância limítrofe, local geográfico de trabalho $(0,05)$ e possuir 3 a 4 animais $(0,05)$, (tabela 4$)$.

\section{DISCUSSÃO}

A freqüência de infecção por leptospira, obtida para a categoria de trabalhadores em Saneamento Ambiental (10,36\%), está entre as mais elevadas das já registradas em inquéritos sorológicos no Brasil. No entanto, essa freqüência é coerente com os valores já obtidos para o grupo estudado (ALMEIDA $\boldsymbol{e t} \boldsymbol{a l} ., \quad 1994 ;$ ANDRADE \& BRANDÃO, 1987).

A variável ocupacional, local geográfico de trabalho, indicada, neste estudo, como fator de risco para a infecção por leptospira, aponta o trabalho no centro da cidade ou em um único bairro como o de menor risco de infecção. A associação da leptospirose com locais geográficos foi registrada por GENDRON et al.(1992); RATHINAM et al. (1993), na Polinésia e na Índia, respectivamente. Tal achado deve estar associado à maior probabilidade de exposição desses trabalhadores a condições ambientais diversificadas, entre estas, as insalubres pois, como afirmam ANDRÉ \& GANIERE (1990), apesar das várias possibilidades na epidemiologia da leptospirose, as condições ambientais são sempre de grande importância.

No ambiente de moradia, houve significância estatística para algumas variáveis relacionadas ao contato com animais e outras com estruturas sanitárias. Desta forma, os indivíduos que possuíam 
Tabela 3 - Fatores de risco (variáveis comportamentais) para infecção por leptospira. Pelotas/RS,

\begin{tabular}{|c|c|c|c|}
\hline Variáveis & $\begin{array}{l}\text { Razão de Odds (bruta) e } \\
\text { IC } 95 \%\end{array}$ & $\begin{array}{l}\text { Número de } \\
\text { Indivíduos }\end{array}$ & $\mathrm{P}$ \\
\hline $\begin{array}{l}\text { Trabalho extra (calhas, } \\
\text { esgotos) }\end{array}$ & & & $=0,4$ \\
\hline Sim & $1,44(0,59-3,50)$ & 32 & \\
\hline Não & 1,00 & 117 & \\
\hline Hábito de caçar, pescar & & & $=0,4$ \\
\hline Sim & $1,43(0,66-3,10)$ & 60 & \\
\hline Não & 1,00 & 89 & \\
\hline Animal sair a rua & & & $=0,1$ \\
\hline Sim & $0,90(0,79-1,02)$ & 75 & \\
\hline Não & 1,00 & 74 & \\
\hline Brincar com o animal & & & $=0,1$ \\
\hline Sim & $1,13(0,49-2,63)$ & 52 & \\
\hline Não & 1,00 & 97 & \\
\hline Tratador do animal & & & $=0,18$ \\
\hline Sim & $2,53(0,87-7,33)$ & 79 & \\
\hline Não & 1,00 & 70 & \\
\hline
\end{tabular}
Brasil. 1991.

caráter social para a leptospirose, vinculada ao saneamento básico. Ou, ainda, com EVERARD et al. (1995), que afirmam não ser a doença inteiramente ocupacional.

A presença de banheiro interno à moradia, como fator de proteção contra a infecção por leptospira, reforça o caráter social da leptospirose, mesmo em um grupo de risco ocupacional. TOMASI et al. (1994) sugerem alguma associação entre tipo de banheiro e diarréia infecciosa em crianças. Assim, pode-se aventar a hipótese de que a presença de banheiros externos à residência ofereceriam maior probabilidade de exposição dos

3 ou 4 animais em casa, apresentaram maior risco de anticorpos antileptospira em relação aqueles que não possuíam animais. Esse achado associa-se, não somente ao número de animais em casa, mas também à presença de outros fatores indiretos, como a espécie animal.

A criação de suínos, no ambiente de moradia dos trabalhadores, como um fator de risco positivo para a infeção por leptospira, ressalta a importância da presença de animais de produção como fator de risco na zona urbana. Neste caso a infecção deve estar muito mais ligada ao contato dos indivíduos com as vias de transmissão contaminadas do que por contato direto com os animais, diversamente do que ocorre com animais de estimação.

CHAPPEL $\boldsymbol{e t}$ al. (1990) incriminaram a espécie suína na transmissão da leptospirose a trabalhadores embora, em sua maioria, associados a criadores profissionais ou moradores na zona rural. A ocorrência de surtos de leptospirose suína e humana foi registrada por GIRIO et al. (1987).

Outro fator de risco associado ao ambiente de moradia dos indivíduos pesquisados, diz respeito à presença de esgotos a céu aberto. É relevante a constatação de que um fator de risco vinculado ao saneamento básico possa estar associado ao local de moradia dos trabalhadores e não ao local de trabalho. Esse achado está de acordo com ANDRADE \& BRANDÃO (1987), que sugerem um indivíduos a locais contaminados por animais, principalmente roedores. JACKSON et al. (1993) sugerem que a presença de condições ambientais favoráveis propiciariam a oportunidade de ocorrência de leptospirose em humanos, inclusive na forma de surtos.

Quanto às variáveis comportamentais avaliadas, não foi possível detectar associação significativa com anticorpos antileptospira. Esse resultado causa certa surpresa, pois KATZ et al.(1997) demonstraram a presença desses fatores na ocorrência de surtos da doença em humanos. Apesar disso, esse achado pode estar relacionado ao tipo de população estudada, trabalhadores em saneamento ambiental, cujos fatores de risco seriam mais específicos.

Os resultados deste estudo, ao ressaltarem a associação entre a infecção por leptospira e a presença de alguns fatores de risco ocupacionais e ambientais no local de moradia dos trabalhadores em saneamento ambiental, confirmam a hipótese da leptospirose não ser uma doença inteiramente ocupacional. Cabendo ainda ressaltar que, embora na última década tenham sobressaídos alguns fatores de risco na epidemiologia da leptospirose, estes associam-se mais a atividades recreativas do que a atividades ocupacionais. O que reforça a possibilidade dos achados deste estudo contribuírem para medidas mais eficazes de prevenção da leptospirose nessa categoria de trabalhadores. 
Tabela 4 - Resultado da regressão logística de fatores de risco ocupacionais e ambientais no local de moradia para infecção por leptospira.

\begin{tabular}{|c|c|c|}
\hline Variáveis & $\begin{array}{c}\text { Razão de odds } \\
\text { (ajustada) e IC } \\
95 \%\end{array}$ & $\begin{array}{l}\text { Nível de Signi- } \\
\text { ficância }\end{array}$ \\
\hline Local geográfico de trabalho & & $\mathrm{p}=0,05$ \\
\hline Centro/único bairro & 1,00 & \\
\hline Conjunto de bairros & $2,04(0,72-5,79)$ & \\
\hline Cidade toda & $2,89(0,97-8,66)$ & \\
\hline Presença de gatos & & $\mathrm{p}=0,4$ \\
\hline Sim & $1,50(0,46-4,81)$ & \\
\hline Não & 1,00 & \\
\hline Lidar com cães & & $\mathrm{p}=0,2$ \\
\hline $\operatorname{Sim}$ & $2,18(0,52-8,98)$ & \\
\hline Não & 1,00 & \\
\hline Lidar com lixo doméstico & & $\mathrm{p}=0,2$ \\
\hline Sim & $1,64(0,67-4,02)$ & \\
\hline Não & 1,00 & \\
\hline Presença de terrenos baldios & & $\mathrm{p}=0,7$ \\
\hline Sim & $1,20(0,33-4,29)$ & \\
\hline Não & 1,00 & \\
\hline Criação de suínos, na moradia & & $\mathrm{p}=0,02$ \\
\hline $\operatorname{Sim}$ & $7,69(1,24-47,63)$ & \\
\hline Não & 1,00 & \\
\hline Número de animais em casa & & $\mathrm{p}=0,05$ \\
\hline Nenhum & 1,00 & \\
\hline $1-2$ animais & $1,41(0,39-5,00)$ & \\
\hline 3-4 animais & $4,70(0,96-22,85)$ & \\
\hline 5 ou mais & $0,88(0,17-4,50)$ & \\
\hline $\mathrm{O}$ banheiro é interno à casa & & $\mathrm{p}=0,02$ \\
\hline Sim & $0,37(0,16-0,85)$ & \\
\hline Não & 1,00 & \\
\hline Presença de esgotos a céu aberto & & $\mathrm{p}=0,04$ \\
\hline Sim & $2,39(1,01-5,64)$ & \\
\hline Não & 1,00 & \\
\hline
\end{tabular}

\section{REFERÊNCIAS BIBLIOGRÁFICAS}

ACHA, P.N., SZYFRES, B. Zoonoses y enfermedades transmisibles comunes al hombre $\mathbf{y}$ a los animales, 2. ed. Washington: OPS, 1986. 989 p. Publicacion Cientifica, 503.

ALMEIDA, L.P., MARTINS, L.F.S., BROD, C.S., et al. Levantamento soroepidemiológico de leptospirose em trabalhadores do serviço de saneamento ambiental em localidade urbana da região sul do Brasil. Rev Saúde Publica, v. 1, n. 28, p. 76-81, 1994.
ALMEIDA, L.P. Estudo retrospectivo de reservatórios animais e vias de transmissão para leptospirose em cinco categorias de trabalhadores. Pelotas - RS, 1991. 126 p. Tese (Mestrado em Sanidade Animal) - Curso de Pós-graduação em Medicina Veterinária, Universidade Federal de Pelotas, 1991.

ANDRADE, J., BRANDÃO, A. Contribuição ao conhecimento da epidemiologia da leptospirose humana, com especial referência ao Grande Rio, Brasil, no período de 1970 a 1982 , Mem Inst Oswaldo Cruz, v. 82, n. 1, p. 91-100, 1987.

ANDRÉ, F., GANIERE, J.P.; New topics on leptospirosis. Comp Immunol Microbiol Infect Dis, v. 13, n. 3, p. 163-168, 1990.

CHAPPEL, R.J., PRIME, R.W., CUTLER, R.S. et al. Antileptospiral antibodies in Australian pig farmers. Med J Aust, v. 152, n. 2, p. 105, 1990.

EVERARD, C.O., EDWARDS, C.N., EVERARD, J.D. et al. A twelve-year study of leptospirosis on Barbados. Eur J Epidemiol, v. 11, n. 3, p. 311-320, 1995.

GENDRON, Y., PRIEUR, J., GAUFROY, X. et al. Les leptospiroses en Polynesie Française: étude de 120 servations. Med Trop, v. 52, n. 1, p. 21-27, 1992.

GIRIO, R.J.S., MATHIAS, L.A, CASTANIA, V.A. $\boldsymbol{e t} \boldsymbol{a l}$. Ocorrência de surtos de leptospirose suína e humana em três propriedades do município de Viradouro, S.P. Cienc Vet, Jaboticabal, v. 1, n. 2, p. 24-25, 1987.

JACKSON, L.A., KAUFMANN, A.F., ADMS, W.G. $\boldsymbol{e}$ t $\boldsymbol{a l}$. Outbreak of leptospirosis associated with swimming. Pediatr Infect Dis, v. 12, n. 1, p. 48-54, 1993.

KATZ, A.R.; SASAKI, D.M.; MUMM, A.H. et al. Leptospirosis on Oahu:na outbreak among military personnel associated with recreational exposure. Mil Med, v. 162, n. 2, p. 101-104, 1997.

KIRKWOOD, B.R. Essentials of medical statistics. Oxford., London, Edinburgh: Blackwell, 1988. 235 p.

MESLIN,F.X. Global aspects of emerging and potential zoonoses: a WHO Perspective. Emerging Infections Diseases, v. 3, n. 2, p. 1-8, 1997.

PERROCHEAU, A., PEROLAT, P. Epidemiology of leptospirosis in New Caledonia South Pacific): a one-year survey. Eur J Epidemiol, v. 13, n. 2, p. 161-167, 1997.

RATHINAM,S.R.; RATHINAM, S.; SELVARAJ,S.; et al. Uveitis associated with na epidemic outbreak of leptospirosis. Am J Ophthalmol, v. 124, n. 1, p. 71-79, 1997.

RATHINAM, S.; EVERARD, C.D.; ALEX, L.C. $\boldsymbol{e}$ t al. Prevalence of leptospiral agglutinins among conservancy workers in Madras city, India. J Trop Hyg, v. 96, n. 1, p. 41-45, 1993.

TOMASI, E.; VICTORA, C.G; POST, P.R.; et al. Uso de chupetas em crianças: contaminação fecal e associação com diarréia. Rev Saúde Pública, v. 28, n. 5, p. 373-379, 1994.

ZARI, S.R., SHIEH, W.J. Leptospirosis associated with outbreak of acute febrile illness and pulmonary haemorrhage, Nicaragua, 1995. Lancet, v. 347, n. 9000, p. 535-536, 1996. 